\title{
Cultura de segurança do paciente em hospitais públicos de ensino: estudo comparativo
}

\author{
Patient safety culture in public teaching hospitals: a comparative study
}

Cultura de seguridad del paciente en hospitales públicos de enseñanza: un estudio comparativo

\author{
Keila Ellen Viana' ๑; Laura Misue Matsuda' ${ }^{\circledR}$; Ana Carolina Simões Pereira' ${ }^{\circledR}$;

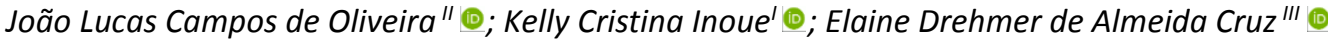

'Universidade Estadual de Maringá, Maringá, PR, Brasil; "Universidade Federal do Rio Grande do Sul, Porto Alegre, RS, Brasil; "'Universidade Federal do Paraná, Curitiba, PR, Brasil

\begin{abstract}
RESUMO
Objetivo: comparar a cultura de segurança do paciente entre equipes de enfermagem de quatro hospitais de ensino públicos do Paraná, Brasil. Método: estudo transversal, descritivo, analítico, realizado em amostra representativa proporcional, aprovado pelo Comitê de Ética em Pequisa. Utilizou-se a versão eletrônica do instrumento Hospital Survey on Patient Safety Culture para a coleta de dados, analisados por meio de estatística descritiva e inferencial, com uso do Software Statistica Single User versão 13. As dimensões da cultura foram classificadas em forte, potencial e frágil; as diferenças foram determinadas pelo teste qui-quadrado e nível de significância $p \leq 0,05$. Resultados: entre os 376 participantes foram constatadas diferenças significativas nas dimensões Frequência de eventos notificados, Percepção de segurança, Abertura para comunicações e Apoio da gerência. Aprendizagem organizacional/melhora continuada foi a única dimensão forte identificada em todos os hospitais. Conclusão: embora observadas diferenças entre hospitais, a cultura de segurança mostra-se frágil e representa barreira à qualidade assistencial.
\end{abstract}

Descritores: Segurança do Paciente; Cultura Organizacional; Hospitais de Ensino; Enfermagem.

\section{ABSTRACT}

Objective: to compare patient safety culture of among nursing teams at four public teaching hospitals in Paraná, Brazil. Method: this cross-sectional, descriptive, analytical study was conducted on a proportional representative sample, and was approved by the Ethics Commission. Data were collected using the electronic version of the Hospital Survey on Patient Safety Culture, and analyzed using descriptive and inferential statistics, using the Statistica Single User software, version 13. The dimensions of culture were classified as strong, potential, and fragile; the differences were determined by the chi-square test to significance level $p<0.05$. Results: significant differences were found among the 376 participants in the following dimensions: Frequency of notified events, Perception of safety, Openness to communications, and Management support. Organizational learning/continuous improvement was the only dimension identified as strong at all the hospitals. Conclusion: although differences were observed among hospitals, safety culture was fragile and constituted a barrier to the quality of nursing care. Descriptors: Patient Safety; Organizational Culture; Hospitals, Teaching; Nursing.

\section{RESUMEN}

Objetivo: comparar la cultura de seguridad del paciente entre los equipos de enfermería de cuatro hospitales públicos de enseñanza en Paraná, Brasil. Método: este estudio transversal, descriptivo y analítico se realizó sobre una muestra representativa proporcional y fue aprobado por la Comisión de Ética. Los datos fueron recolectados usando la versión electrónica de la Encuesta Hospitalaria sobre Cultura de Seguridad del Paciente, y analizados usando estadística descriptiva e inferencial, usando el software Statistica Single User, versión 13. Las dimensiones de cultura fueron clasificadas como fuerte, potencial y frágil; las diferencias se determinaron mediante la prueba de chi-cuadrado al nivel de significancia $p<0.05$. Resultados: se encontraron diferencias significativas entre los 376 participantes en las siguientes dimensiones: Frecuencia de eventos notificados, Percepción de seguridad, Apertura a las comunicaciones y Apoyo a la gerencia. El aprendizaje organizacional / mejora continua fue la única dimensión identificada como fuerte en todos los hospitales. Conclusión: si bien se observaron diferencias entre hospitales, la cultura de seguridad fue frágil y constituyó una barrera para la calidad de la atención de enfermería.

Descriptores: Seguridad del Paciente; Cultura Organizacional; Hospitales de Enseñanza; Enfermería.

\section{INTRODUÇÃO}

No cenário da segurança do paciente - elemento indissociável da qualidade do cuidado - os eventos adversos (EA) se evidenciam nos serviços de saúde e; portanto, são considerados problema de saúde pública mundial ${ }^{1}$. Devido a periculosidade própria da assistência em saúde, na qual, mesmo que esforços rumo às barreiras de segurança sejam exponenciais (principalmente nos países desenvolvidos), a ocorrência de erros e EA ainda é realidade inegável, principalmente no ambiente hospitalar².

Uma pesquisa realizada em oito hospitais na Irlanda constatou prevalência de $12,2 \%$ de EA e destes, mais de $70 \%$ foram considerados evitáveis ${ }^{3}$. No Brasil, dados recentes do Sistema Único de Saúde apontam que de março de 2014 a junho de 2019 ocorreram 331.136 EA, 93,3\% em ambiente hospitalar ${ }^{4}$. 
Com intuito de fomentar a implementação de estratégias voltadas à segurança do paciente, desde 2013 o Programa Nacional de Segurança do Paciente determina medidas com vistas a qualificar os cuidados ofertados no serviço de saúde em seus três níveis de atenção e tem como uma das frentes elementares o desenvolvimento da cultura de segurança do paciente (CSP) $)^{5,6}$.

A CSP consiste em uma série de habilidades, comportamentos, valores e atitudes que se relacionam ao comprometimento individual e coletivo com a gestão e a melhoria da segurança nos serviços de saúde ofertados ${ }^{5}$.

Portanto, a adoção desse "aparato institucional”, eminentemente cultural, é importante porque possibilita a mobilização de esforços para que os pontos considerados frágeis sejam solucionados e aqueles considerados favoráveis, fortalecidos ${ }^{6}$. Em outras palavras, a CSP pode ser barreira ou facilitador para que as estratégicas de segurança do paciente ocorram na dinâmica da prestação dos cuidados em saúde ${ }^{7}$.

$\mathrm{Na}$ avaliação da CSP como uma etapa chave para o incremento de estratégias factíveis ao cuidado seguro, pesquisas recentes realizadas na Croácia, Omã, Turquia, Malásia e Brasil apontam cultura punitiva relacionada aos erros; inadequação do número de pessoal/trabalhadores; elevado estresse no ambiente de trabalho e; necessidade de desenvolvimento de ações que transponham o trabalho seguro prescrito, sugerindo que existem demandas à melhorias $^{8-12}$.

As dificuldades/barreiras referidas são expressas comumente por enfermeiros, atores reconhecidamente estratégicos nos processos de planejamento e execução de ações de segurança do paciente ${ }^{7}$. É evidente, portanto, a importância de se identificar e comparar dados referentes à CSP relacionados à enfermagem porque, ações desse tipo podem fomentar estratégias de melhorias na dinâmica de trabalho da equipe de saúde, com mais assertividade e qualidade.

Considerando que a enfermagem é, inegavelmente, protagonista no processo de assistência à saúde e na melhoria de indicadores da qualidade da assistência, este estudo se pauta na seguinte questão: No que se refere à avaliação da segurança do paciente, existe diferença entre as equipes de enfermagem de hospitais públicos de ensino de um mesmo estado? Para responder a essa indagação o presente estudo tem como objetivo comparar a cultura de segurança do paciente entre equipes de enfermagem de quatro hospitais de ensino públicos do Paraná, Brasil.

\section{MÉTODO}

Estudo transversal, descritivo e analítico, realizado no período de junho a setembro de 2019, em quatro hospitais de ensino públicos do estado do Paraná, Brasil, denominados Hospital A; Hospital B; Hospital C e; Hospital D. Essas instituições possuem número de leitos que variam de 123 a 313.

Os participantes da pesquisa foram profissionais da equipe de enfermagem e que obedeceram aos seguintes critérios de inclusão: trabalhar em um dos hospitais do estudo, independentemente do vínculo empregatício (servidor público estatutário, celetista, credenciado, ou prestador de serviço) ou área de atuação, por no mínimo três meses. Foram excluídos aqueles que, por qualquer motivo, estivessem afastados do trabalho durante o período de coleta de dados ou que não responderam ao instrumento de coleta de dados na íntegra.

O tamanho da amostra foi estabelecido por meio de amostragem representativa proporcional com a totalidade ( $\mathrm{N}=1.969)$ de profissionais de enfermagem dos hospitais pesquisados, utilizando-se o programa StatDisk versão 8.4. Para isto, adotou-se nível de confiança de $95 \%$, erro amostral de $5 \%$ e proporção populacional de $50 \%$, cujo resultado ( $n=322$ ) acrescido de 15\% para reposição de perdas, determinou a amostra mínima de 371 participantes, estratificada entre os locais de estudo.

Para a coleta de dados utilizou-se o E-Questionário de Cultura de Segurança Hospitalar, que consiste numa alternativa eletrônica/digital, validada, do Hospital Survey on Patient Safety Culture (HSOPSC). Esse questionário é constituído por 8 seções ( $\mathrm{A}$ à $\mathrm{H}$ ) agrupadas em 12 dimensões e 61 perguntas/itens, acrescidas de dois itens: "Número de notificações de incidentes" e "Nota geral da Segurança do Paciente", os quais não compõem as dimensões da CSP e são avaliados de forma separada. Os itens se apresentam agrupados por temas, de acordo com as 12 dimensões e as respostas são dadas na forma de escala do tipo Likert em 5 níveis ${ }^{13}$.

Ressalta-se que a seção " $G$ " do instrumento se destina à caracterização dos participantes da pesquisa e avaliação da segurança do paciente no hospital, por mensuração em escala de 10 pontos. Já a seção H, item opcional, fornece espaço para comentários acerca da segurança do paciente ${ }^{13}$.

A análise da CSP é realizada com base no percentual de respostas positivas atribuídas a cada dimensão e classificadas em: Área forte - ou positiva - para a segurança do paciente (75\% ou mais de respostas positivas); Área com potencial - ou neutra - para a segurança do paciente ( $50 \%$ a $74,9 \%$ de respostas positivas) e; Área frágil - ou negativa - para a segurança do paciente $(49,9 \% \text { ou menos de respostas positivas })^{14}$. 
Previamente à coleta de dados, foi solicitada autorização aos responsáveis técnicos dos Serviços de Enfermagem das instituições. Os dados foram coletados de forma presencial, em horário de trabalho, por meio de visitas agendadas aos setores.

Os dados foram digitados em planilhas do Microsoft Office Excel ${ }^{\oplus}$. Após, foram transportados e analisadas no software Statistica Single User versão 13, por meio de técnicas estatísticas descritiva e inferencial. As variáveis categóricas foram analisadas por medidas de proporção percentual. Para testar a diferença (comparação) das dimensões entre os hospitais utilizou-se o teste qui-quadrado, adotando-se o nível de significância de $5 \%$, expresso em p-valor $\leq 0,05$

O projeto de pesquisa deste estudo foi submetido à apreciação do Comitê de Ética em Pesquisas institucionalizado na Plataforma Brasil e aprovado sob o número do parecer 3.480.841/2019 e CAAE 12854419.2.0000.0104 em 04/09/2019.

\section{RESULTADOS E DISCUSSÃo}

Participaram do estudo 376 profissionais, caracterizados conforme consta na Tabela 1.

TABELA 1: Distribuição da equipe de enfermagem de hospitais de ensino públicos do Paraná, de acordo com a categoria profissional e setor ( $n=376)$. Paraná, Brasil, 2019.

\begin{tabular}{llllll}
\hline Categorias & $\begin{array}{c}\text { Hospital A } \\
\mathbf{n}(\%)\end{array}$ & $\begin{array}{c}\text { Hospital B } \\
\mathbf{n}(\%)\end{array}$ & $\begin{array}{c}\text { Hospital C } \\
\mathbf{n}(\%)\end{array}$ & $\begin{array}{c}\text { Hospital D } \\
\mathbf{n}(\%)\end{array}$ & $\begin{array}{l}\text { Total } \\
\mathbf{n}(\%)\end{array}$ \\
\hline $\begin{array}{l}\text { Categoria Profissional } \\
\text { Enfermeiro }\end{array}$ & $27(23,3)$ & $26(30,2)$ & $43(38,4)$ & $27(43,5)$ & $123(32,7)$ \\
Técnico de Enfermagem & $89(76,7)$ & $56(65,1)$ & $69(61,6)$ & $29(46,8)$ & $243(64,6)$ \\
Auxiliar de Enfermagem & - & $4(4,7)$ & - & $6(9,7)$ & $10(2,7)$ \\
Setor & & & & & \\
Cirúrgico & $28(24,1)$ & $6(7,0)$ & $18(16,1)$ & $14(22,6)$ & $66(17,6)$ \\
Clínico & $12(10,3)$ & $8(9,3)$ & $17(15,2)$ & $13(21,0)$ & $50(13,3)$ \\
Pediatria & $4(3,4)$ & $14(16,3)$ & $7(6,3)$ & $8(12,9)$ & $33(8,8)$ \\
UTI & $34(29,3)$ & $28(32,6)$ & $38(33,9)$ & $16(25,8)$ & $116(30,9)$ \\
Obstetrícia & $28(24,1)$ & $6(7,0)$ & $6(5,4)$ & $9(14,5)$ & $49(13,0)$ \\
Emergência & $4(3,4)$ & $18(20,9)$ & $15(13,4)$ & - & - \\
Outros & $6(5,2)$ & $6(7,0)$ & $11(9,8)$ & $2(3,2)$ & $25(6,6)$ \\
\hline
\end{tabular}

Analisando, comparativamente, constata-se que houve predominância da categoria técnicos de enfermagem (64,6\%), atuantes em Unidades de Terapia Intensiva (UTI) (30,9\%).

Na Tabela 2 constam os dados referentes aos percentuais de respostas positivas, neutras e negativas, das 12 dimensões da cultura de segurança do paciente, atribuídas pelas equipes de enfermagem dos quatro hospitais.

A análise das dimensões da CSP entre os hospitais considerou a média e o erro padrão das respostas positivas, que resultaram em diferenças estatísticas significativas para quatro dimensões: Frequência de eventos notificados $(p=0,0221)$; Percepção de segurança $(p=0,0129)$; Abertura para comunicações $(p=0,0023)$ e; Apoio da gerência do hospital para a segurança do paciente $(p=0,0040)$. Esses dados indicam que as dimensões referidas possuem diferentes avaliações da cultura de segurança pelos profissionais de enfermagem entre os hospitais. Esse fato pode estar relacionado ao tempo da implantação das estratégias de segurança do paciente nas instituições pesquisadas, ou também porque os profissionais percebem essas dimensões como sendo as mais deficitárias.

Na dimensão frequência de eventos notificados, pode-se verificar que o percentual de respostas positivas nos hospitais B $(29,1 \%)$ e C $(43,8 \%)$ apresentam indicador significativamente menor do que os hospitais A (50,9\%) e D $(50,0 \%)$, que embora tenham obtido melhores resultados, não foram suficientes para classificar a dimensão como fortalecida. Esse resultado pode estar relacionado com a recente implantação do Núcleo de Segurança do Paciente em todos os hospitais investigados, ou ainda, à cultura de culpabilização que, de acordo com a literatura, acarreta prejuízo ao sistema e gera subnotificações de $E A^{15}$.

A culpabilização individual é o que mais inibe os profissionais a apontarem os erros, desencadeando a subnotificação6,10,15. O medo que os profissionais possuem acerca da punição pode estimular a subnotificação, resultando na dificuldade institucional em mensurar a qualidade do cuidado ofertado e em proporcionar estratégias de melhorias em voga à segurança do paciente ${ }^{8,15}$. 
TABELA 2: Comparação da cultura de segurança do paciente entre hospitais de ensino públicos por profissionais de enfermagem ( $n=376)$. Paraná, Brasil, 2019.

\begin{tabular}{|c|c|c|c|c|c|c|}
\hline Dimensões & $\begin{array}{c}\text { Hospital A } \\
\begin{array}{c}\text { (n=116) } \\
n(\%)\end{array}\end{array}$ & $\begin{array}{c}\text { Hospital B } \\
\begin{array}{c}(n=86) \\
n(\%)\end{array}\end{array}$ & $\begin{array}{c}\text { Hospital C } \\
\text { (n=112) } \\
n(\%)\end{array}$ & $\begin{array}{c}\text { Hospital D } \\
\begin{array}{c}(n=62) \\
n(\%)\end{array}\end{array}$ & $\begin{array}{c}\text { Total } \\
(n=376) \\
n(\%)\end{array}$ & p-valor \\
\hline \multicolumn{7}{|c|}{ D1 - Frequência de eventos notificados } \\
\hline Positiva & $59(50,9)$ & $25(29,1)$ & $49(43,8)$ & $31(50,0)$ & $164(43,6)$ & \multirow{3}{*}{$0,0221^{*}$} \\
\hline Negativa & $42(36,2)$ & $39(45,3)$ & $47(42,0)$ & $18(29,0)$ & $146(38,8)$ & \\
\hline Neutra & $15(12,9)$ & $22(25,6)$ & $16(14,3)$ & $13(21,0)$ & $66(17,6)$ & \\
\hline \multicolumn{7}{|c|}{ D2 - Percepção de segurança } \\
\hline Positiva & $16(13,8)$ & $7(8,1)$ & $26(23,2)$ & $10(16,1)$ & $59(15,7)$ & \multirow{3}{*}{$0,0129 *$} \\
\hline Negativa & $83(71,6)$ & $70(81,4)$ & $64(57,1)$ & $46(74,2)$ & $263(69,9)$ & \\
\hline Neutra & $17(14,7)$ & $9(10,5)$ & $22(19,6)$ & $6(9,7)$ & $54(14,4)$ & \\
\hline \multicolumn{7}{|c|}{ D3 - Expectativas e ações da direção/supervisão da unidade/serviço } \\
\hline Positiva & $21(18,1)$ & $20(23,3)$ & $21(18,8)$ & $16(25,8)$ & $78(20,7)$ & \multirow{3}{*}{0,2764} \\
\hline Negativa & $67(57,8)$ & $51(59,3)$ & $64(57,1)$ & $26(41,9)$ & $208(55,3)$ & \\
\hline Neutra & $28(24,1)$ & $15(17,4)$ & $27(24,1)$ & $20(32,3)$ & $90(23,9)$ & \\
\hline \multicolumn{7}{|c|}{ D4 - Aprendizagem organizacional/melhora continuada } \\
\hline Positiva & $102(87,9)$ & $74(86,0)$ & $96(85,7)$ & $47(75,8)$ & $319(84,8)$ & \multirow{3}{*}{0,4144} \\
\hline Negativa & $9(7,8)$ & $6(7,0)$ & $7(6,3)$ & $8(12,9)$ & $30(8,0)$ & \\
\hline Neutra & $5(4,3)$ & $6(7,0)$ & $9(8,0)$ & $7(11,3)$ & $27(7,2)$ & \\
\hline \multicolumn{7}{|c|}{ D5 - Trabalho em equipe na unidade/serviço } \\
\hline Positiva & $80(69,0)$ & $58(67,4)$ & $72(64,3)$ & $46(74,2)$ & $256(68,1)$ & \multirow{3}{*}{0,3266} \\
\hline Negativa & $34(29,3)$ & $23(26,7)$ & $38(33,9)$ & $13(21,0)$ & $108(28,7)$ & \\
\hline Neutra & $2(1,7)$ & $5(5,8)$ & $2(1,8)$ & $3(4,8)$ & $12(3,2)$ & \\
\hline \multicolumn{7}{|c|}{ D6 - Abertura para comunicações } \\
\hline Positiva & $65(56,0)$ & $40(46,5)$ & $61(54,5)$ & $25(40,3)$ & $191(50,8)$ & \multirow{3}{*}{$0,0023^{*}$} \\
\hline Negativa & $43(37,1)$ & $29(33,7)$ & $30(26,8)$ & $17(27,4)$ & $119(31,6)$ & \\
\hline Neutra & $8(6,9)$ & $17(19,8)$ & $21(18,8)$ & $20(32,3)$ & $66(17,6)$ & \\
\hline \multicolumn{7}{|c|}{ D7 - Feedback e comunicação sobre erros } \\
\hline Positiva & $83(71,6)$ & $58(67,4)$ & $75(67,0)$ & $38(61,3)$ & $254(67,6)$ & \multirow{3}{*}{0,2731} \\
\hline Negativa & $23(19,8)$ & $14(16,3)$ & $25(22,3)$ & $11(17,7)$ & $73(19,4)$ & \\
\hline Neutra & $10(8,6)$ & $14(16,3)$ & $12(10,7)$ & $13(21,0)$ & $49(13,0)$ & \\
\hline \multicolumn{7}{|c|}{ D8 - Resposta não punitivas para erros } \\
\hline Positiva & $78(67,2)$ & $49(57,0)$ & $64(57,1)$ & $44(71,0)$ & $235(62,5)$ & \multirow{3}{*}{0,3154} \\
\hline Negativa & $29(25,0)$ & $31(36,0)$ & $40(35,7)$ & $13(21,0)$ & $113(30,1)$ & \\
\hline Neutra & $9(7,8)$ & $6(7,0)$ & $8(7,1)$ & $5(8,1)$ & $28(7,4)$ & \\
\hline \multicolumn{7}{|c|}{ D9 - Dimensionamento de pessoal } \\
\hline Positiva & $23(19,8)$ & $14(16,3)$ & $23(20,5)$ & $22(35,5)$ & $82(21,8)$ & \multirow{3}{*}{0,0589} \\
\hline Negativa & $71(61,2)$ & $59(68,6)$ & $75(67,0)$ & $28(45,2)$ & $233(62,0)$ & \\
\hline Neutra & $22(19,0)$ & $13(15,1)$ & $14(12,5)$ & $12(19,4)$ & $61(16,2)$ & \\
\hline \multicolumn{7}{|c|}{ D10 - Apoio da gerência do hospital para a segurança do paciente } \\
\hline Positiva & $14(12,1)$ & $22(25,6)$ & $34(30,4)$ & $10(16,1)$ & $80(21,3)$ & \multirow{2}{*}{$0,0040^{*}$} \\
\hline Negativa & $102(87,9)$ & $64(74,4)$ & $78(69,6)$ & $52(83,9)$ & $296(78,7)$ & \\
\hline \multicolumn{7}{|c|}{ D11 - Trabalho em equipe } \\
\hline Positiva & $42(36,2)$ & $23(26,7)$ & $31(27,7)$ & $20(32,3)$ & $116(30,9)$ & \multirow{3}{*}{0,6305} \\
\hline Negativa & $48(41,4)$ & $47(54,7)$ & $56(50,0)$ & $29(46,8)$ & $180(47,9)$ & \\
\hline Neutra & $26(22,4)$ & $16(18,6)$ & $25(22,3)$ & $13(21,0)$ & $80(21,3)$ & \\
\hline D12 - Transf & interna e pas & sagem de pla & ntão & & & \\
\hline Positiva & $4(3,4)$ & $4(4,7)$ & $12(10,7)$ & $1(1,6)$ & $21(5,6)$ & \\
\hline Negativa & $106(91,4)$ & $77(89,5)$ & $99(88,4)$ & $58(93,5)$ & $340(90,4)$ & 0,0582 \\
\hline Neutra & $6(5,2)$ & $5(5,8)$ & $1(0,9)$ & $3(4,8)$ & $15(4,0)$ & \\
\hline
\end{tabular}

* Teste qui-quadrado significativo

Uma pesquisa recente de revisão da literatura, com objetivo de identificar e analisar publicações nacionais sobre motivos para a não notificação de incidentes de segurança do paciente por profissionais, no contexto dos serviços de saúde brasileiros, evidenciou os principais motivos: medo ou receio em notificar; a notificação era focada somente em eventos mais graves; falta de conhecimento sobre o tema ou como notificar e; centralização da notificação no profissional enfermeiro ${ }^{15}$. 
A literatura explanada, somada aos achados deste estudo, permitem identificar que a subnotificação de falhas é uma realidade problemática e isso, certamente se atrela no fato de ser uma prática que demanda impulso pessoal para informar sobre os incidentes, mas também necessita de apoio institucional para enfrentar questões que muitas vezes são tabus na dinâmica do trabalho.

Quanto à percepção de segurança, em relação ao percentual de respostas positivas, observa-se que os hospitais A $(13,8 \%)$ e $B(8,1 \%)$ obtiveram indicador significativamente menor aos hospitais C $(23,2 \%)$ e $D(16,1 \%)$. Esse fato pode estar associado a dificuldade dos profissionais em identificar ações institucionais voltadas à cultura de segurança, aliada a não consolidação dessa nas instituições. Em cenários assim, concorda-se com a literatura quando indica como importante a adoção de estratégias institucionais para a sensibilização dos colaboradores e a aproximação das lideranças com a equipe assistencial ${ }^{16}$.

No tocante às dificuldades para a implantação da cultura de segurança em instituições de saúde, constata-se que temas da cultura organizacional são compreendidos como obstáculo da gestão e podem se intensificar no âmbito hospitalar porque, de acordo com a literatura ${ }^{17}$, as características históricas dos serviços tendem a ser desfavoráveis às mudanças e podem interferir na aquisição de novos hábitos institucionais.

$\mathrm{Na}$ dimensão abertura para as comunicações, os percentuais de respostas positivas apontam diferença significativa, pois os hospitais A $(56,0 \%)$ e C $(54,5 \%)$ apresentam indicador significativo maior em relação aos hospitais B $(46,5 \%)$ e D $(40,3 \%)$, sugerindo que aqueles possuem a cultura de comunicação melhor instituída e que existe maior abertura para o diálogo referente aos erros. No âmbito da comunicação, para que haja melhoria nos indicadores da qualidade do cuidado é necessário que as categorias profissionais sejam estimuladas a comunicar erros, visto que a abertura para a comunicação é um meio eficaz ao fortalecimento da CSP7,15,17.

Esse fato, referente à abertura para comunicação, é importante porque, a facilidade na transmissão de informações entre diferentes níveis hierárquicos na organização possibilita o desenvolvimento do trabalho em equipe, e repercute na maior adesão dos trabalhadores ao processo de notificação de $E A^{18}$.

Em relação ao apoio da gerência do hospital para a segurança do paciente, os dados exibiram diferenças importantes no percentual de respostas positivas, pois os hospitais $A(12,1 \%)$ e $D(16,1 \%)$ apresentaram indicador significativamente menor em relação aos hospitais $B(25,6 \%)$ e $C(30,4)$. Os resultados desta dimensão sugerem que há falta de apoio da gerência à implementação de estratégias para a segurança do paciente nas instituições. Aspecto esse abordado também num estudo ${ }^{7}$ que menciona o fato de hospitais de ensino públicos possuírem apoio deficitário para aplicar as estratégias de segurança que são agravadas por mudanças no contexto político e pouca flexibilidade gerencial, devido a necessidade de se cumprir as leis que regem a Administração Pública.

Num ambiente em que há falta de apoio institucional, evidencia-se a importância do comprometimento dos gestores em criar estratégias de segurança do paciente porque, tais práticas proporcionam assistência segura e, por isso, devem ser incentivadas por meio de políticas institucionais sistêmicas e contínuas ${ }^{7}$.

No que diz respeito à avaliação individual das dimensões pelas equipes, por hospital, em cultura forte, potencial ou frágil para a segurança do paciente, os resultados demonstram que os quatro hospitais apresentaram somente uma dimensão forte, ou seja, a dimensão aprendizagem organizacional/melhora continuada. Já os hospitais que obtiveram maior número de dimensões no quesito, com potencial para a segurança, foi o $A$ (cinco) seguido do $C$ (quatro), $D$ (quatro), e B (três). Por fim, o hospital que apresentou o maior número de dimensões frágeis para a segurança foi o Hospital B (oito), seguido do C (sete), D (sete), e A (seis).

Os dados referidos, coadunam uma pesquisa comparativa ${ }^{19}$ realizada no Hospital Universitário do Rio de Janeiro (Brasil) e em Porto (Portugal), a qual constatou que somete uma dimensão foi considerada forte para a segurança no Hospital português e, nenhuma no Hospital brasileiro. Dentre às dimensões que se apresentaram frágeis para a segurança, o Hospital do Brasil obteve número maior (oito) que o Hospital de Portugal (cinco).

Outro estudo, realizado em um hospital de ensino de Curitiba-PR, não identificou dimensão forte para a segurança; quatro dimensões foram consideradas como potencial e oito foram frágeis ${ }^{20}$. De acordo com a literatura, evidências assim reforçam a necessidade de se investir na criação e melhorias de estratégias que atuem em prol dos processos de segurança do paciente ${ }^{12}$.

A comparação entre as instituições investigadas, à luz dos trabalhadores de enfermagem, apresenta o panorama geral da Cultura de Segurança proporcionando ferramentas sólidas para a gestão da segurança nas instituições hospitalares.

\section{Limitações do estudo}

No que se refere à limitação deste estudo, a amostragem não aleatória pode ser apontada como fator interveniente. Entretanto, considerando que o estado do Paraná possui sete hospitais de ensino públicos e a presente 
investigação abarcou quatro, é possível que a realidade constatada reflita, de fato, o situação da CSP nesse tipo de instituições hospitalar, à luz da equipe de enfermagem.

\section{CONCLUSÃO}

Quatro dimensões da cultura de segurança do paciente demonstraram diferença com significância estatística. Destas, frequência de eventos notificados e abertura para comunicações foram consideradas com potencial para a segurança em dois hospitais e frágil nos outros dois.

A única dimensão considerada forte para a segurança do paciente, em todos os hospitais, foi a aprendizagem organizacional/melhora continuada. Os resultados mostram a fragilidade da CSP na equipe de enfermagem e isto, certamente, pode comprometer a qualidade assistencial.

Os resultados possibilitam afirmar que a comparação realizada entre as instituições mostra o panorama geral de como se apresenta a Cultura de Segurança, do ponto de vista dos trabalhadores da enfermagem, o que, sem dúvida é uma base de evidências importante para o (re)planejamento de ações de segurança na assistência hospitalar pública do Estado.

A própria comparação realizada também é uma possível contribuição do estudo, no sentido de que a prática/cultura de empregar o benchmarking entre serviços públicos (algo ainda incomum) seja fomentada.

\section{REFERÊNCIAS}

1. Montserrat-Capella D, Suarez M, Ortiz L, Mira JJ, Duarte HG, Reveiz L, et al. Frequency of ambulatory care adverse events in Latin American countries: the AMBEAS/PAHO cohort study. Int. J. Qual. Health Care [Internet], 2015 [cited 2019 Oct 10]; 27(1):52-9. DOI: https://doi.org/10.1093/intqhc/mzu100

2. Grossmann N, Gratwohl F, Musy SN, Nielen NM, Simon M, Donzé J. Describing adverse events in medical inpatients using the Global Trigger Tool. Swiss Med Wkly [Internet], 2019 [cited 2019 Oct 10]; 149:w20149. DOI: https://doi.org/10.4414/smw.2019.20149

3. Rafter N, Hickey A, Conroy RM, et al. The Irish National Adverse Events Study (INAES): the frequency and nature of adverse events in Irish hospitals: a retrospective record review study. BMJ Qual Saf. [Internet], 2017 [cited 2019 Oct 17]; $26(2): 111-9$. DOI: http://dx.doi.org/10.1136/bmjqs-2015-004828

4. Brasil. Ministério da Saúde (BR). Agência Nacional de Vigilância Sanitária. Boletim de segurança do paciente e qualidade em serviços de saúde: incidentes relacionados à saúde assistência à saúde - 2018. Brasília (DF) [Internet], 2018 [cited 2019 Nov 17 ]. Available from:

http://portal.anvisa.gov.br/documents/33852/3074203/Boletim+Seguran\%C3\%A7a+do+Paciente+e+Qualidade+em+Servi\%C3\% A7os+de+Sa\%C3\%BAde+n+20+-+Incidentes+Relacionados+\%C3\%A0+Assist\%C3\%AAncia+\%C3\%A0+Sa\%C3\%BAde++2018/094a8d6a-d6eb-4d90-9bc2-49047f4c2fe3

5. Brasil. Ministério da Saúde (BR). Portaria no 529/2013, 1 de abril de 2013. Institui o programa nacional de segurança do paciente. Brasília (DF) [Internet], 2013 [cited 2019 Nov 20]. Available from: https://bvsms.saude.gov.br/bvs/saudelegis/gm/2013/prt0529_01_04_2013.html

6. Abreu IM, Rocha RC, Avelino FVSD, Guimarães DBO, Nogueira LT, Madeira MZA. Patient safety culture at a surgical center: the nursing perception. Rev. Gaúcha Enferm. [Internet], 2019 [cited 2019 Nov 10]; 40(esp):e20180198. DOI: http://dx.doi.org/10.1590/1983-1447.2019.20180198

7. Reis GAX, Oliveira JLC, Ferreira AMD, Marcon SS, Matsuda LM. Difficulties to implement patient safety strategies: perspectives of management nurses. Rev Gaúcha Enferm. [Internet], 2019 [cited 2019 Nov 15]; 40(esp):e20180366. DOI: https://doi.org/10.1590/1983-1447.2019.20180366

8. Brborović O, Brborović H, Nola IA, Milošević M. Culture of Blame-An Ongoing Burden for Doctors and Patient Safety. Int. J. Environ. Res. Public. Health [Internet], 2019 [cited 2019 Nov 16]; 16(23):4826. DOI: https://doi.org/10.3390/ijerph16234826

9. Al Nadabi W, Faisal M, Mohammed MA. Patient safety culture in Oman: A national study. J. Eval. Clin. Pract. [Internet], 2019 [cited 2019 Dec 19]; 26(5):1406-15. DOI: https://doi.org/10.1111/jep.13322

10. Teles M, Kaya S. Staff perceptions of patient safety culture in general surgery departments in Turkey. Afr. Health Sci. [Internet], 2019 [cited 2019 Dec 19]; 19(2):2208-18. DOI: https://doi.org/10.4314/ahs.v19i2.46

11. Alex Kim RJ, Chin ZH, Sharlyn P, Priscilla B, Josephine S. Hospital survey on patient safety culture in Sarawak General Hospital: A cross sectional study. Med. J. Malaysia [Internet], 2019 [cited 2019 Nov 24]; 74(5):385-8. Available from: https://pubmed.ncbi.nlm.nih.gov/31649213/

12. Siman AG, Braga LM, Amaro MOF, Brito MJM. Practice challenges in patient safety. Rev. Bras. Enferm. [Internet], 2019 [cited 2019 dec 20]; 72(6):1504-11. DOI: http://dx.doi.org/10.1590/0034-7167-2018-0441

13. Andrade LELA, Melo LOM, Silva IG, Souza RM, Lima ALB, Freitas MR, et al. Adaptation and validation of the Hospital Survey on Patient Safety Culture in an electronic Brazilian version. Epidemiol. Serv. Saude [Internet], 2017 [cited 2019 Nov 22]; 26(3):45568. DOI: http://dx.doi.org/10.5123/s1679-49742017000300004

14. Sorra JS, Nieva VF. Hospital survey on patient safety culture. Rockville: Agency for Healthcare Research and Quality [Internet], 2004 [cited 2019 Nov 13]; Available from: http://www.ahrq.gov/professionals/qualitypatientsafety/patientsafetyculture/hospital/userguide/hospcult.pdf 
15. Alves MFT, Carvalho DS, Albuquerque GSC. Barriers to patient safety incident reporting by Brazilian health professionals: an integrative review. Ciênc. saúde coletiva [Internet], 2019 [cited 2019 apr 01]; 24(8):2895-908. DOI: https://doi.org/10.1590/1413-81232018248.23912017

16. Magalhaes FHL, Pereira ICA, Luiz RB, Barbosa MH, Ferreira MBG. Patient safety atmosphere in a teaching hospital. Rev. Gaúcha Enferm. [Internet], 2019 [cited 2020 mar 26]; 40 (esp): e20180272. DOI: https://doi.org/10.1590/1983-1447.2019.20180272

17. Oliveira JLC, Matsuda LM. Benefits and difficulties in the implementation of hospital accreditation: The voice of quality managers. Esc. Anna Nery [Internet], 2016 [cited 2019 Oct 16]; 20(1):63-9, 2016. Available from: http://www.redalyc.org/articulo.oa?id=127744318009

18. Alswat K, Abdalla RAM, Titi MA, Bakash M, Mehmood F, Zubairi, et al. Improving patient safety culture in Saudi Arabia (20122015): trending, improvement and benchmarking. BMC Health Serv. Res. [Internet], 2017 [cited 2019 Nov 26]; 17(1):516. DOI: https://doi.org/10.1186/s12913-017-2461-3

19. Fassarella CS, Camerini FG, Henrique DM, Almeida LF, Figueiredo MCB. Evaluation of patient safety culture: comparative study in university hospitals. Rev. esc. enferm. USP [Internet], 2018 [cited 2019 Nov 25]; 52:e03379. DOI: http://dx.doi.org/10.1590/s1980-220x2017033803379

20. Cruz EDA, Rocha DJM, Mauricio AB, Ulbrich FS, Batista J, Maziero ECS. Safety culture among health professionals in a teaching hospital. Cogitare Enferm. [Internet], 2018 [cited 2019 Nov 25]; 23 (1): e50717. DOI: http://dx.doi.org/10.5380/ce.v23i1.50717. 